\title{
Spatial Correlation in Uniform Circular Arrays Subject to Mutual Coupling
}

\author{
Hendrik Rogier, Postdoctoral Fellow FWO-V. \\ Information Technology Department, Ghent University \\ St.-Pietersnieuwstraat 41, B-9000 Ghent, Belgium. \\ E-mail: hendrik.rogier@intec.UGent.be, \\ Tel.: $++32 / 92643343$ Fax: $++32 / 92649969$.
}

\section{Introduction}

The diversity performance of the multi-antenna system depends heavily on the signal correlations between the different antenna elements. Strongly correlated antenna signals substantially reduce the diversity gain of the receiver. In absence of mutual coupling, the spatial correlation can be easily evaluated. In the presence of mutual coupling, however, the treatment becomes more involved and the complex pattern correlation differs substantially from the theoretical estimate of the envelope correlation [1], mainly because of the deformation of the antenna pattern due to mutual coupling. In new communication systems, based on adaptive and MIMO antennas, antenna correlation also has a major influence on the performance and mutual coupling is playing an important role. In $[2,3]$, a rigorous model describing mutual coupling in uniform circular arrays (UCAs) was presented based on a spherical-mode expansion. This model is extended here for the analysis of the signal correlation between the antenna elements in UCAs. The performance of UCAs in diversity systems was already analyzed in [4], where the monopoles were assumed to be minimum scattering antennas, having a sinusoidal current distribution. Here, we will follow a more general approach. In Section 2 the spherical-modes technique introduced in [2] is extended to UCAs with arbitrary oriented antenna elements (in [2] the antennas were assumed to carry only vertical currents). This framework is then applied to calculate the spatial correlation for an UCA given a Laplacian energy distribution in the azimuth plane in Section 3. Our analysis includes all mutual coupling effects of the UCA. In [3] the effect of antenna tilt on signal correlation was investigated. Here, we analyze the signal correlation as a function of frequency, given a nine-element UCA of dipoles.

\section{Spherical-modes based circuit model}

Consider a general uniform circular array (UCA), for which the parameters of the circuit model in transmit and receive mode are known. In a first step, the circular symmetry is exploited by expanding the radiation patterns $\mathbf{F}_{i}(\phi, \theta)=$ $\left(F_{i, \phi}(\phi, \theta), F_{i, \theta}(\phi, \theta)\right)$ into a Fourier series of phase modes in the azimuth variable: $\mathbf{F}_{i}(\phi, \theta)=\sum_{m=-\infty}^{+\infty} \mathbf{F}_{i, m}^{\phi}(\theta) e^{j m \phi}$. Then, once we know the series expansion for a certain port $i$, we construct the radiation pattern at an arbitrary port $j$ by relying on symmetry $\mathbf{F}_{j}(\theta, \phi)=\mathbf{F}_{i}\left(\theta, \phi-\phi_{j}+\phi_{i}\right)=\sum_{m=-M}^{+M} \mathbf{F}_{i, m}^{\phi}(\theta) e^{j m\left(\phi-\phi_{j}+\phi_{i}\right)}$. The

same procedure applied to the impedance matrix reduces $\overline{\bar{Z}}$ to the phase-sequence impedance $Z_{m}^{\phi}$ for each phase mode $m$, given by $Z_{m}^{\phi}=\sum_{n=1}^{N} Z_{i, n} e^{-j \frac{2 \pi(i-n)}{N}}$, with 
$Z_{i, n}$ a matrix element of $\overline{\bar{Z}}$ and $N$ the number of array elements. Given the symmetry in the phase modes $\mathbf{F}_{i, m}^{\phi}(\theta)$ we focus on one particular antenna port of the array and also drop the subscript there, so we continue the discussion with $\mathbf{F}_{m}^{\phi}(\theta)$. The summation can be restricted to $m$ running from $-M$ to $M$, provided that $M \gg k_{0} d$, with $d$ the largest dimension of the array [2]. For the dependence of the radiation pattern on the continuous parameter $\theta$, we propose an expansion into vector spherical modes for the radiation vector $\mathbf{F}_{m}^{\phi}(\theta)$. In this expansion, the $\theta$-dependence of $F_{\phi, m}^{\phi}(\theta)$ and $F_{\theta, m}^{\phi}(\theta)$ is expressed as

$$
\begin{gathered}
F_{\phi, m}^{\phi}(\theta)=j \sum_{n=1}^{+\infty}\left[A_{n, m}^{\phi, \theta} \frac{m P_{n}^{|m|}(\cos \theta)}{\sin \theta}+B_{n, m}^{\phi, \theta} \frac{d P_{n}^{|m|}(\cos \theta)}{d \theta}\right] \\
F_{\theta, m}^{\phi}(\theta)=\sum_{n=1}^{+\infty}\left[A_{n, m}^{\phi, \theta} \frac{d P_{n}^{|m|}(\cos \theta)}{d \theta}+B_{n, m}^{\phi, \theta} \frac{m P_{n}^{|m|}(\cos \theta)}{\sin \theta}\right] .
\end{gathered}
$$

The open-circuit voltages at the different antenna-elements due to a horizontally polarized and a vertically polarized plane wave impinging on the array are related to $F_{\phi, m}^{\phi}(\theta)$ and $F_{\theta, m}^{\phi}(\theta)$, respectively. Details are given in [3].

\section{The spatial correlation given a Laplacian distribution}

Consider the UCA being illuminated by a vertically polarized incoming signal with a Laplacian DOA distribution $f(\phi)$ in the azimuth plane $\left(\theta=90^{\circ}\right)$ :

$$
f(\phi)=\frac{a}{2\left(1-e^{-a \pi}\right)} e^{-a|\xi-\phi|}, \quad-\pi+\xi<\phi<\pi+\xi,
$$

with $\xi$ the main direction of the scattering cluster and $a$ a measure for the angle spread. For all antenna ports terminated by a load $Z_{0}$, the spatial variance between ports $i$ and $j$ is given by:

$$
\sigma_{s}^{2}(i, j)=\sum_{m, n=-M}^{+M} \frac{a^{2}\left|Z_{0}\right|^{2} V_{0, m}^{\mathrm{V}, \phi} e^{j m\left(\xi-\phi_{i}\right)} V_{0, n}^{* \mathrm{~V}, \phi} e^{-j n\left(\xi-\phi_{j}\right)}}{\left(1-e^{-a \pi}\right)\left(Z_{0}+Z_{m}^{\phi}\right)\left(Z_{0}^{*}+Z_{n}^{* \phi}\right)} \frac{1-(-1)^{m-n} e^{-a \pi}}{a^{2}+(m-n)^{2}} .
$$

where we used the shorthand notation

$$
\begin{aligned}
V_{0, m}^{\mathrm{V}, \phi}=V_{0, m}^{\mathrm{V}, \phi}\left(\theta=90^{\circ}\right)= & -\frac{2 j \lambda}{R_{c}} \sum_{l=0}^{+\infty} \frac{(-1)^{|m|+l+1}}{2^{l} l !}[2(|m|+l)-1] ! ! \\
& \times\left[[2(|m|+l)+1] A_{|m|+2 l+1, m}^{\phi, \theta}-m B_{|m|+2 l, m}^{\phi, \theta}\right],
\end{aligned}
$$

with $n ! !=1.3 .5 \ldots(n-2) . n$ for $n$ odd. The spatial correlation is then given by $R_{s}(i, j)=\frac{\sigma_{s}^{2}(i, j)}{\sigma_{s}(i, i) \sigma_{s}(j, j)}$. In absence of mutual coupling, the spatial correlation for an UCA given a Laplacian energy distribution reduces to the result given in [5]:

$$
\sigma_{s}^{2}(i, j)=\sum_{l=-\infty}^{+\infty} \frac{(-1)^{l} a^{2} e^{j l\left(\xi-\frac{\phi_{i}+\phi_{j}}{2}\right)}}{\left(1-e^{-a \pi}\right)} \frac{1-(-1)^{l} e^{-a \pi}}{a^{2}+l^{2}} J_{l}\left(2 k_{0} r \sin \theta\left|\sin \left(\frac{\phi_{i}-\phi_{j}}{2}\right)\right|\right)
$$

For $a \rightarrow 0$ the Laplacian distribution becomes a uniform distribution in the azimuth plane. More details are found in [3], together with the expressions for the spatial correlation given a uniform distribution over the complete unit sphere. 


\section{Example: Nine-element array of dipoles}

Consider a nine-element array of thin dipole antennas tuned to $900 \mathrm{MHz}$ (dipole length $l=16.12 \mathrm{~cm}$. The elements are distributed uniformly on a circle with diameter

$d=l\left(\approx \frac{\lambda}{2}\right.$ at $\left.\left.900 \mathrm{MHz}\right)\right)$. Figs. 1 shows the correlation between antenna elements 1 and 2, 1 and 3,1 and 4, 1 and 5, respectively, for a cluster located at $\theta=45^{\circ}$ with $a=3$ (see (2)). The analytical calculation based on the theory in Section 3 is validated by means of a Monte-Carlo simulation using an ensemble of 400000 pseudo-random DOAs, distributed following the same Laplacian distribution. When the antenna elements are loaded $\left(Z_{0}=73 \Omega\right)$, one notices a substantial decrease in the correlation at $900 \mathrm{MHz}$, for all antenna element combinations considered. The reason why can be seen in Fig 2, where we plotted the measured voltage at port 1 as a function of the azimuth angle of the incoming plane wave. The pattern is near to omnidirectional when all ports are open-circuited. However, when the ports are loaded with $Z_{0}=73 \Omega$, the pattern becomes directional with a main beam along $0^{\circ}$. Now there are no significant contributions from side-lobes. When increasing the frequency, the correlation curve tends to oscillate around the curve for which mutual coupling was neglected. When the antenna ports are open circuited, the correlation oscillates very rapidly in the region around $1800 \mathrm{MHz}$, because at $1800 \mathrm{MHz}$ the antenna elements now form a lossless array structure that can be seen as consisting of two vertically stacked arrays consisting of resonating shortcircuited $\frac{\lambda}{2}$ elements. Nearly all elements are good field receptors in this case and high EM-field concentrations build up in the complete array structure. These fields are damped by adding loads at the different antenna ports.

\section{References}

[1] C. B. Dietrich, K. Dietze, J. R. Nealy, and W. L. Stutzman, "Spatial, polarization, and pattern diversity for wireless handheld terminals," IEEE Trans. Antennas Propag., vol. 49, pp. 1271-1281, Sep 2001.

[2] H. Rogier and E. Bonek, "Analytical spherical-mode based compensation of mutual coupling in uniform circular arrays for direction-of-arrival estimation," Int. Journal of Electr. and Comm., vol. 60, pp. 179-189, Mar. 2006.

[3] H. Rogier, "Spatial correlation in uniform circular arrays based on a sphericalmodes model for mutual coupling," Int. Journal of Electr. and Comm., vol. 60, pp. 521-532, Jul. 2006.

[4] R. G. Vaughan, J. Bach Andersen, and M. H. Langhorn, "Circular array of outward sloping monopoles for vehicular diversity antennas," IEEE Trans. Antennas Propag., vol. 36, pp. 1365-1374, Oct 1988.

[5] J.-A. Tsai, M. Buehrer, and B. D. Woerner, "Spatial fading correlation function of circular antenna arrays with Laplacian energy distribution," IEEE Communications Letters, vol. 6, pp. 178-180, May 2002. 

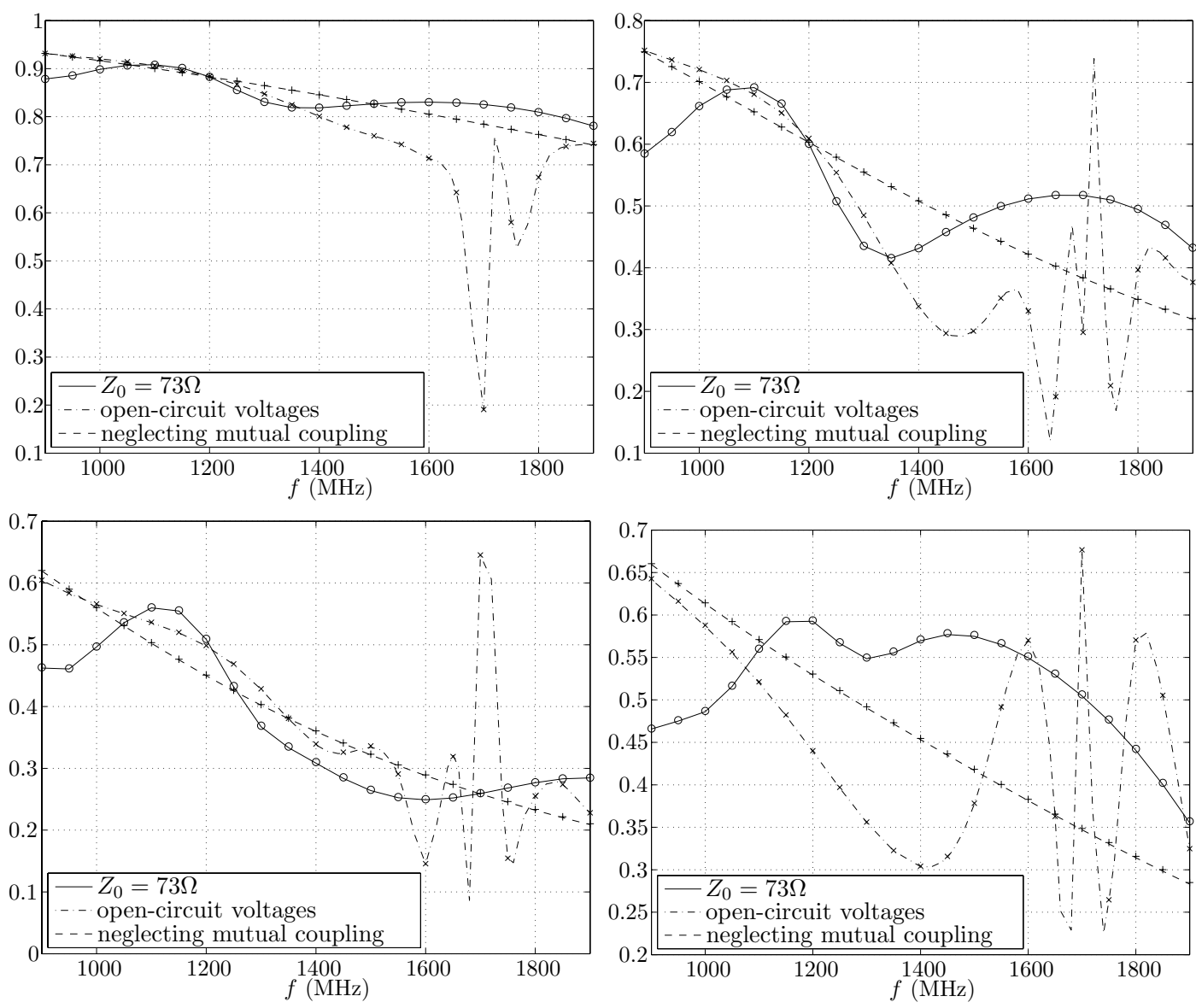

Figure 1: Correlation between antenna elements 1 and 2 (top left), 1 and 3 (top right), 1 and 4 (bottom left), and 1 and 5 (bottom right). Monte-Carlo simulation results: $(+)$ : neglecting mutual coupling, $(\times)$ : open-circuit voltages, $(\circ): Z_{0}=73 \Omega$

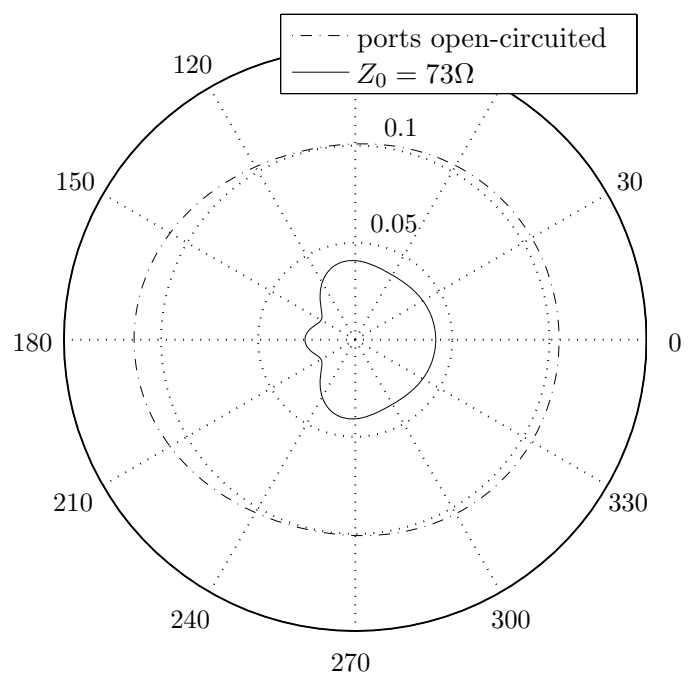

Figure 2: Measured voltage at port 1 for $f=900 \mathrm{MHz}$. 


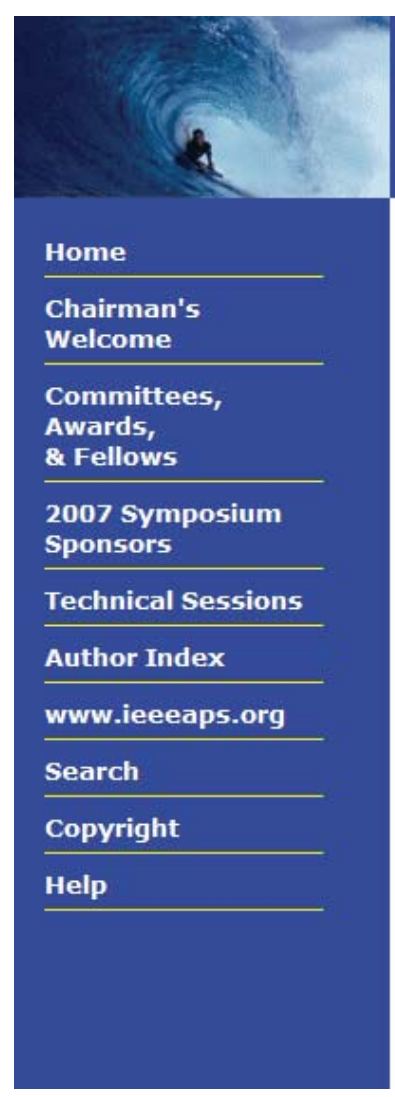

2007 IEEE Antennas and Propagation Society International Symposium

Sheraton Waikiki | Honolulu, Hawaii, USA | June 10-15, 2007
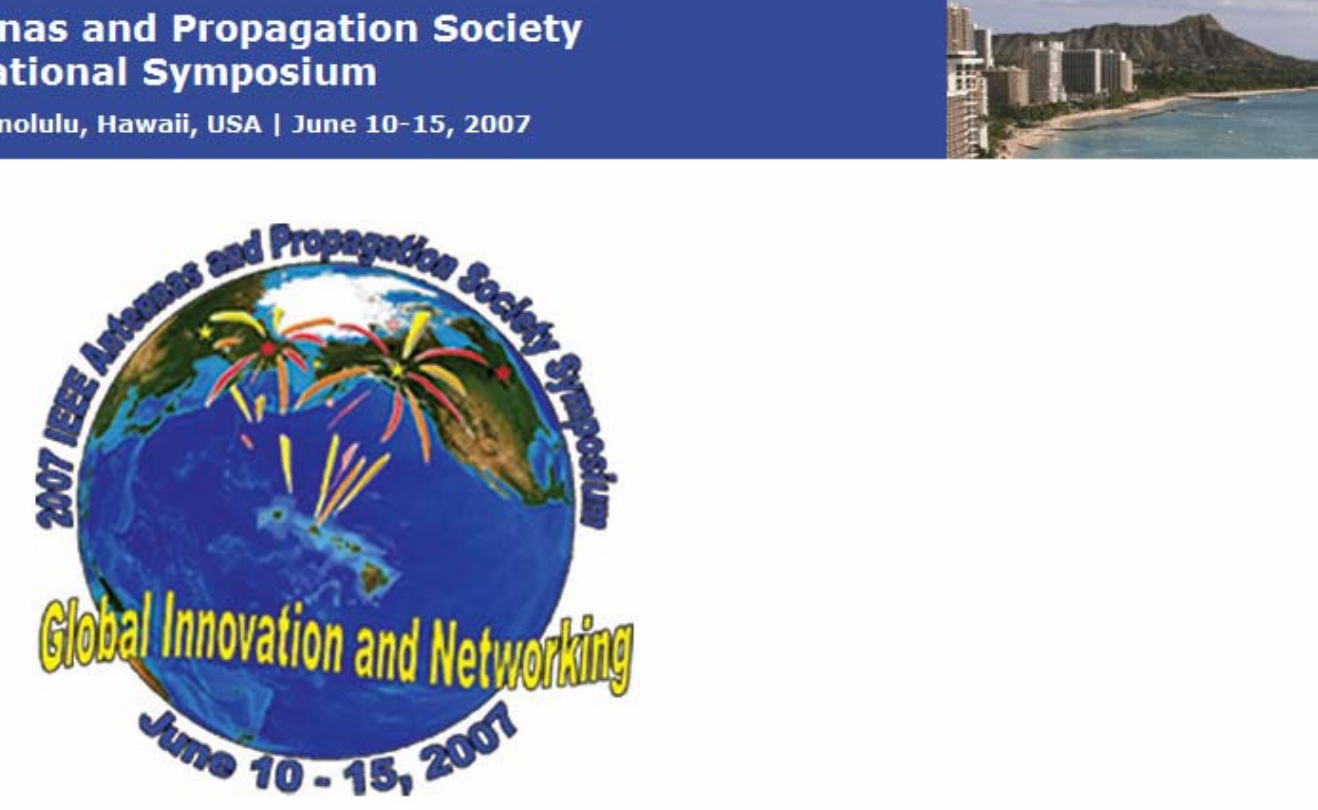

\section{- IEEE}

$(A / 5)$ 